\title{
Dynamic Deformation Using Adaptable, Linked Asynchronous FEM Regions
}

\author{
Umut Koçak* Karljohan Lundin Palmerius ${ }^{\dagger} \quad$ Matthew Cooper \\ Norrköping Visualization and Interaction Studio, Department of Science and Technology, \\ Linkoping University, SWEDEN
}

\begin{abstract}
In order to simulate both physically and visually realistic soft tissue deformations, the Finite Element Method (FEM) is the most popular choice in the literature. However it is non-trivial to model complex behaviour of soft tissue with sufficient refresh rates, especially for haptic force feedback which requires an update rate of the order of $1 \mathrm{kHz}$. In this study the use of asynchronous regions is proposed to speed up the solution of FEM equations in real-time. In this way it is possible to solve the local neighborhood of the contact with high refresh rates, while evaluating the more distant regions at lower frequencies, saving computational power to model complex behaviour within the contact area. Solution of the different regions using different methods is also possible. To attain maximum efficiency the size of the regions can be changed, in real-time, in response to the size of the deformation.
\end{abstract}

Keywords: Finite Element Method, surgery simulation, haptics

\section{Introduction}

The simulation of deformable soft tissues with physically realistic force feedback in real time is still one of the big challenges in the haptics area. The major challenge is the computational burden to simulate realistic deformations which cannot be easily handled using even today's fastest computers. Therefore a compromise between realism and speed has to be achieved in the simulation of soft tissue deformation. There are two general methods applied in the simulations: mass-spring and finite element models. Sufficient refresh rates can be reached by using mass-spring models; however the physical properties of materials cannot be taken into account in these methods. Although studies reveal that it is possible to simulate physically realistic deformations by using Finite Element Methods(FEM), the real-time requirement is not easy to satisfy because of the complexity of this approach. Soft tissues may also have various complex behaviours like visco-elasticity and anisotropicity, in addition to non-linearity. If tissue cutting is also to be simulated, the model should be updated both physically and visually, which makes the real-time simulations even harder.

To reach a compromise between the necessity of high haptic refresh rates and the computational burden, several optimization techniques such as condensation, pre-computation, level of detail, and exploitation of the sparse matrix structure have been introduced.

\footnotetext{
*e-mail: umut.kocak@itn.liu.se

†e-mail:karljohan.palmerius@itn.liu.se

†e-mail:matthew.cooper@itn.liu.se
}

Current simulators, however, still have to either sacrifice one property of real tissues such as non-linearity, anisotropicity or viscoelasticity, or apply force interpolation or extrapolation techniques to reach a sufficient haptic refresh rate $(1 \mathrm{kHz})$. Therefore solution of large FEM systems in real-time to be used in soft-tissue deformations is still a challenge for which new solutions are being sought.

In this study, a technique analogous to the application of level of detail is proposed but using the time domain instead of the spatial domain. This approach has been named asynchronous regions. The nodes of the FEM model are separated into different regions, each of which can be solved with different frequencies and by different solution techniques and the size of which are adaptable in realtime. The idea aims to compute in the local neigbourhood around the contact node with a high frequency while calculating the more remote nodes with lower frequencies. Since the local neigbourhood around the contact is both visually and physically more significant than the more distant regions, more computation power can thus be dedicated to either implementing non-linearity, visco-elasticity, anisotropicity or increasing the resolution in the local neigbourhood. In order to study the effect of the use of the asynchronous regions, our experiments are designed to solve the whole model with a frequency of $1 \mathrm{kHz}$ and compare the characteristics of the response with those of the solution using asynchronous regions. Therefore a one dimensional model is used so that it is possible to analytically solve the model at $1 \mathrm{kHz}$ with a sufficient number of nodes in one dimension to test various sizes of regions. The output force and the modification of the model are recorded by applying different input signals to the model to illustrate the effects of asyncronous regions.

The outline of the paper is as follows: the second section summarizes the previous work using FEM in soft tissue deformation. The model and different solution methods are described in the third and fourth sections respectively. The fifth section introduces the asynchronous and adaptable asynchronous regions. The results are presented in the sixth section and finally conclusions and future work are discussed.

\section{Related Work}

There are several research groups working on the simulation of deformable objects. FEM is not the only method used for simulations but it is considered to achieve the most physically realistic deformations. In this section a range of studies are summarized, most of which address the problem of haptic force feedback using FEM for real-time applications.

Linear FEM is used in the studies [Frank et al. 2001; Mor and Kanade 2000; Nienhuys and van der Stappen 2000; Lindblad and Turkiyyah 2007; Sela et al. 2007; Wu and Heng 2005]. In [Sela et al. 2007], FEM calculations are done offline during the preprocessing step to evaluate constraints to simulate tissue cutting, then the constraints for the nodes along the cutting path are moved and the model is updated by using discontinuous discrete free form deformations during the simulation. In [Wu and Heng 2005] modification of the tissue cutting was permitted but to achieve this the possible operation area is restricted to a local region in the prepocessing step. The others [Lindblad and Turkiyyah 2007; Mor and Kanade 
2000; Nienhuys and van der Stappen 2000] also implemented tissue cutting, however all of these studies use linear equations which are not appropriate for soft tissues.

To achieve higher realism, non-linear and anisotropic tissues are modeled in [Picinbono et al. 2003]. Since the calculations for nonlinear models take longer, the linear displacement equations are solved for the whole model, and non-linear components are added only for a local neighbourhood of the contact area. To simulate larger deformations realistically, instead of just squeezing or poking the model, non-linear equations for the strain are used in [Zhuang and Canny 2000].

Pre-processing, which restricts the functionality of the simulation in most cases, or an optimization method is needed for solving nonlinear systems. The idea of recording the response of the system for all nodes to a unit input in pre-processing is implemeted in [Cotin et al. 1999; Sedef et al. 2006]. [Sedef et al. 2006] exploited the idea to model linear visco-elasticity. The same principle is used together with local dynamic regions in a hybrid model to allow cutting the soft tissue in [Delingette et al. 1999], which restricts the cutting region depending on specific surgery types in the pre-calculation step. The hybrid model with a restricted operation region and adaptive meshes are implemented in [Heng et al. 2004] and [Wu et al. 2001]. The common problem in these studies is the limitation of the preprocessing making real-time updates either impossible or restricted to a pre-specified local region. Extended FEM (XFEM), which allows discontinuities in the model, is exploited to update the model faster in case of tissue cutting in [Vigneron et al. 2004]. Other optimizations like condensation[Bro-Nielsen and Cotin 1996], use of graded meshes[Yan et al. 2007], force extrapolation[Picinbono et al. 2000] and computation on the GPU[Wu and Heng 2005] are proposed for solution of FEM equations.

Most of the studies above are lacking either one or more of nonlinearity, visco-elasticity, or real-time modification features. Furthermore none of them has achieved a sufficient haptic refresh rate $(1 \mathrm{kHz})$. Therefore a different technique has to be applied in the field to meet the requirements. The idea of updating different regions of a model with different frequencies is mentioned in [Cavusoglu and Tendick 2000] for spring models and in [Astley and Hayward 1998; Debunne et al. 2001] for FEM. However none of these FEM studies compare the response of local regions with the whole solution or discuss the implementation details. The aim of this study is to make a comprehensive discussion and prove the concept of asynchronous regions, allowing the evaluation of a realistic force at $1 \mathrm{kHz}$, as an alternative optimization technique in FEM based applications. Therefore visco-elasticity and non-linearity has not been considered yet. However, application of the asynchronous regions depends on the same principles presented throughout this paper, for the case of non-linear and visco-elastic models.

\section{Model}

A linear FEM model is created by using the basic principles of finite elements[Zienkiewicz et al. 2005]. Material properties like elasticity of modulus, damping and mass can be set as well as geometric properties for each element individually. The element stiffness matrix is computed by evaulating integral (1) over the volumetric region $\Omega$. B describes the element geometry and $\mathbf{D}$ is a material matrix which depends on the elasticity of modulus and poisson ratio. After the computation of the element stiffness matrices, the global stiffness matrix for the whole model is formed( See [Zienkiewicz et al. 2005] for further details ). To keep the model stationary in the scene, boundary conditions are defined at least for one node. Before the simulation starts, the stiffness matrix is condensed such that it does not contain any entries for the boundary condition nodes while preserving their effect.

$$
\mathbf{K}=\int_{\Omega} \mathbf{B}^{\mathrm{T}} \mathbf{D} \mathbf{B} d \Omega
$$

In the case of dynamic equations, the distributed mass is approximated with concentrated masses by diagonalizing the mass matrix which results in better performance. By using a diagonal mass and damping matrix it is possible to solve the system as independent linear equations in the case of explicit dynamic solution systems.

After the calculation of the material properties, the boundary conditioned nodes are constrained to stay constant. A collision algorithm is used to choose the closest node of the model to the haptic probe's position as a contact node when the probe is within a reasonable neighborhood of the model specified by a threshold value. A force input is applied to the contact node by using a spring model between the haptic probe and the current position of the contact node. The stiffness constant of this spring model should be chosen to reach a compromise between steady state and transient response errors as discussed in [Barbagli et al. 2003]. A much greater constant than the stiffness of the deformable model results in oscillations in force, while a much lower one causes high steady state errors resulting in a large position difference between the haptic probe and the contact node. The output force of this spring model is then applied as input to the FEM model to calculate the new displacements of the nodes including the contact node. At the end of the haptic frame the force sent back to the haptic device is calculated by the spring model using the updated position of the contact node.

\section{FEM Solver}

Different solvers including explicit dynamic, semi-implicit dynamic and static solvers have been implemented to solve the FEM equations. However to achieve realistic deformation behaviour, only dynamic solvers are used in the simulation. The general formula for dynamic solutions is;

$$
\mathbf{M u}+\mathbf{C u}+\mathbf{K u}=\mathbf{f}
$$

in which the stiffness matrix, displacements, forces, mass and damping are represented by $\mathbf{K}, \mathbf{u}, \mathbf{f}, \mathbf{M}$ and $\mathbf{C}$ respectively while $\dot{\mathbf{u}}$ and $\ddot{\mathbf{u}}$ refer to velocity and acceleration.

The simulation time is discretized into time-steps to evaluate numerical integration of the solution and the unknowns are solved at each time step. There are two general ways of solving the discretized system: Implicit and Explicit methods.

In implicit methods the unknown values at time $(t+1)$ are used to evaluate the values at time $(t+1)$. To solve the equations either a linear system of equations has to be solved or a sparse matrix inversion has to be used. Therefore, if the stiffness matrix changes (for example in non-linear systems or in tissue cutting) it is not possible to achieve realistic interactive rates with implicit methods. However these methods are unconditionally stable such that the convergence is guaranteed even for large timesteps $(\Delta t)$. A semi-implicit method is implemented in this study by using the formula,

$$
\hat{\mathbf{K}} \mathbf{u}(t+\Delta t)=\hat{\mathbf{f}}(t)
$$




$$
\begin{aligned}
\hat{\mathbf{K}} & =\frac{\mathbf{M}}{\Delta t^{2}}+\frac{\mathbf{C}}{2 \Delta t}+\mathbf{K} \\
\hat{\mathbf{f}}(t) & =\frac{2 \mathbf{M}}{\Delta t^{2}} \mathbf{u}(t)+\left(\frac{\mathbf{C}}{2 \Delta t}-\frac{\mathbf{M}}{\Delta t^{2}}\right) \mathbf{u}(t-\Delta t)+\mathbf{f}(t)
\end{aligned}
$$

The $\hat{\mathbf{K}}$ and $\hat{\mathbf{f}}$ need to be updated in each frame since $\Delta \mathrm{t}$ is not constant between two frames, then (3) is solved by either Gaussian Elimination or Conjugate Gradient method.

In explicit solutions the known values at time $t$ are used to calculate the values at time $t+1$. Unlike the implicit methods the differential equations can be written independently if lumped mass and damping is used. Therefore explicit methods are not only faster but also make it easier to make changes in the stiffness matrix in real time. Nevertheless explicit methods are only conditionally stable such that the time step $(\Delta \mathrm{t})$ used in the calculations must be less than a critical time $\left(\Delta \mathrm{t}_{\text {critical }}\right) . \Delta \mathrm{t}_{\text {critical }}$ is often formulized as:

$$
\Delta t_{\text {critical }} \approx l \sqrt{\frac{\rho}{E}}
$$

$E, l$ and $\rho$ represent elasticity, minimum finite element length in the model, and mass density respectively in (4). Experiments show, however, that the damping coefficient also has an effect on $\Delta \mathrm{t}_{\text {critical }}$ as discussed in [Delingette et al. 1999]. Because of the restriction on the time step, explicit methods can only be used for soft tissues. The need to have smaller time-steps also results in larger relaxation and convergence times. In this study two different explicit methods are used. The first is a finite difference Euler method with the equation (5) where $\mathbf{m}$ and $\mathbf{c}$ refer to mass and damping respectively.

$$
\begin{aligned}
\mathbf{u}(t+\Delta t)=\mathbf{u}(t) & +\frac{2-\mathbf{c} \Delta t}{2+\mathbf{c} \Delta t}(\mathbf{u}(t)-\mathbf{u}(t-\Delta t)) \\
& -\frac{2 \Delta t^{2}}{\mathbf{m}(2+\mathbf{c} \Delta t)}(\mathbf{K u}(t)-\mathbf{f}(t))
\end{aligned}
$$

The second method (6) is a combination of an implicit and explicit Euler method which is;

$$
\begin{aligned}
\mathbf{u}(t+\Delta t) & =\mathbf{u}(t)+\Delta t \dot{\mathbf{u}}(t+\Delta t) \\
\dot{\mathbf{u}}(t+\Delta t) & =\dot{\mathbf{u}}(t)+\Delta t \ddot{\mathbf{u}}(t) \\
\ddot{\mathbf{u}}(t) & =\frac{\mathbf{f}(t)-\mathbf{c m} \dot{\mathbf{u}}(t)-\mathbf{K u}(t)}{\mathbf{m}}
\end{aligned}
$$

The use of asynchronous regions allows choosing different solvers described above for different regions. While solving the equations for the current active region, the values of the nodes in other regions are used without knowing the solver type of those regions. The solvers for different regions are chosen before the simulation starts. The only restriction is the instability condition, explained above, for explicit solvers. To be able to use sufficient resolution and meet the stability requirement (4), the refresh rates for the explicit solvers have to be kept high. Therefore for the regions with low refresh rates, the semi-implicit solver (3) should be chosen while the explicit solvers can be chosen for the regions with higher frequencies. A detailed disscussion on the comparison of the implicit and explicit methods is presented in [Bro-Nielsen and Cotin 1996].

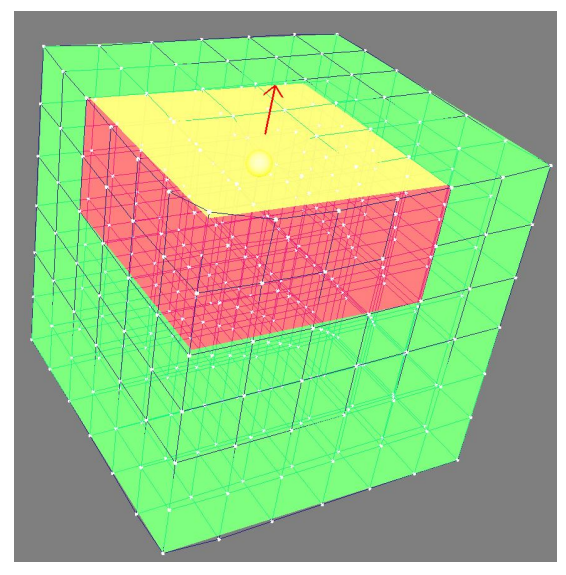

Figure 1: The asynchronous regions in 3D updated in real-time.

\section{Asynchronous Regions}

The biggest challenge of using FEM in tissue deformation is the computational burden since sufficient resolution to model the tissue results in large numbers of equations that must be solved in realtime. In the case of surgery simulations, in which cutting the soft tissue is simulated, fulfilling both realism and refresh rate requirements becomes particularly problematic for these complex models. Therefore aynchronous regions are proposed in this study to optimize the solution of the FEM equations in real time while preserving the physical response of the modelled system.

\subsection{Regions}

The asynchronous regions are the separate parts of the model where the corresponding FEM equations are solved with different refresh rates. To create the regions, the FEM solution is divided into different groups each of which is then solved with different frequencies. This feature is exploited by solving the displacements of the nodes in the local neighbourhood of the contact node (called the primary region) with a high frequency and the other regions (the secondary regions) with lower frequency. It is also possible to choose different solution methods for each region from the ones described in the previous section, which allows the use of explicit dynamic methods, which are suitable only for soft tissues because of their instability, for soft tissue while other methods are used for hard tissues.

The boundaries for an asynchronous region are kept in a vector of a border structure which keeps the beginning and ending node indices of a subset of the region. This is illustrated in figure 2 in $1 \mathrm{D}$ and 2D. The red nodes represent the primary region which is solved with $1 \mathrm{kHz}$, while the green and blue nodes refer to secondary regions which are solved with $100 \mathrm{~Hz}$ and $10 \mathrm{~Hz}$ respectively for this instance. The node indices for the members of borders vector for the green region are illustrated in figure 2(b). The green region is represented by a vector of borders; $\{(42,48),(54,55),(59,60),(67,68),(72,73),(80,81)$, $(85,86),(93,99)\}$. The same approach is used for 3 dimensional case to create the regions' border vectors. The connection nodes between two different regions cause the regions to be affected by each other enabling the deformation behaviour to traverse throughout the model even though the regions are updated with different frequencies.

At the beginning of the simulation the number of asynchronous re- 


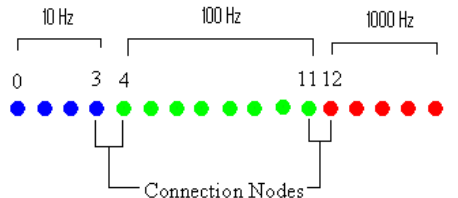

(a) 1 dimension

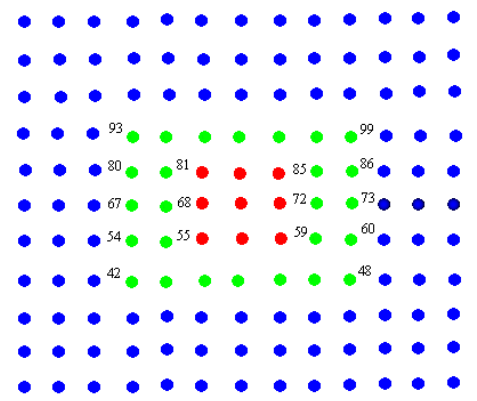

(b) 2 dimensions

Figure 2: The allocation of asynchronous regions on the model.

gions and the size and refresh rate of each are set to default values. In the event of a contact the boundaries for the asynchronous regions are set depending on the contact node and the sizes of the regions, and they are updated only when the contact node changes or the region sizes are adapted in response to the changing contact, as explained in subsection 5.5. Figure 1 shows a screen dump from the simulation when the asynchronous regions are rendered with different colors.

\subsection{Solving Equations}

At the beginning of each haptic loop the current region is chosen depending on the refresh rate parameters. After the current region is chosen, the input in that frame is applied to the model and only the displacements of the nodes in the current region are solved.

In the case of static or semi-implicit methods, in which writing the FEM equations independently is not possible, the FEM equations need to be reorganized before solving the system for the current region. For all rows corresponding to the nodes in the active region, the displacements of the nodes in the other regions are multiplied with corresponding matrix coefficients, and the sum of these multiplications are subtracted from the force value for that row as formulized below;

$$
\mathbf{f}_{i_{\text {modified }}}=\mathbf{f}_{i}-\sum_{j \notin R_{a}} \mathbf{K}_{i j} \mathbf{u}_{j}, \forall i \in R_{a}
$$

where $R_{a}$ refers to the active region which is being solved in the current frame.

The allocation of the asynchronous regions in the stiffness matrix is illustrated in figure 3 by using the same colors in figure 2 . The non-zero values are shown by dashed lines and the $j_{t h}$ indices in (7) refer to the white spaces for each row. Since the stiffness matrix has a banded structure, which is exploited in the evaluation of (7), the nodes in a region are affected only by the displacement of the connection nodes (shown in figure 2(a) for 1D) of the neigbouring regions. It can be observed that the active regions have some

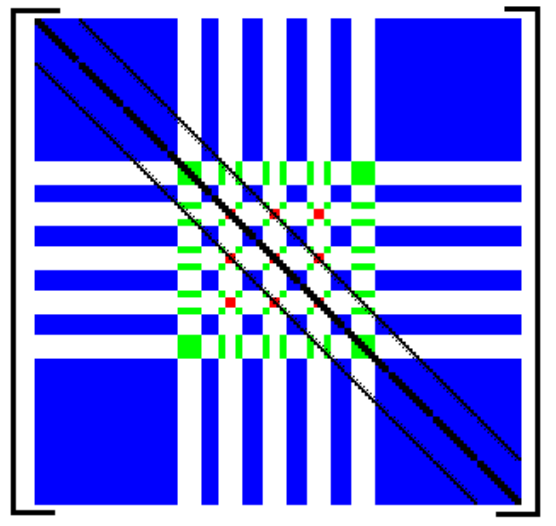

Figure 3: The asynchronous regions in the solution matrix.

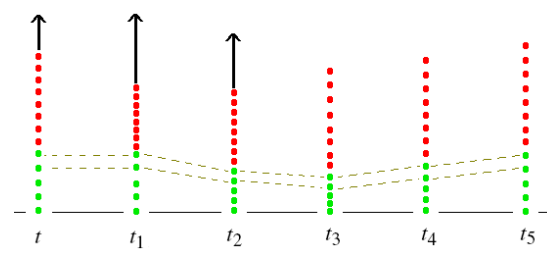

Figure 4: The use of interpolated values for the low frequency regions

non-zero values in the columns corresponding to the connection nodes of their neighbour regions in the rows corresponding to their own connection nodes. After the application of (7) to preserve a relationship between connection nodes, a copy of the stiffness matrix containing only the entries of the active region is created to be solved in the regular way.

For explicit dynamic simulations the independent equations are solved only for the nodes in the current region without the need to embed the effect of the connection nodes to the other side. After the system is solved the displacements are updated locally and the output force on the contact node is evaluated.

\subsection{Interpolation}

Should the refresh rates for the secondary regions be too low, these regions behave like a moving wall such that when the nodes in the primary region are evaluated the nodes of the secondary regions stay constant. Then when the nodes in the secondary regions are solved the nodes will have a sudden jump in displacement values since the displacements of the primary region nodes have been evaluated many times and may have very large displacement changes between the time step of the secondary regions in case of continuous input. This 'moving wall' effect will cause discontinuity in both haptic and graphic rendering and will also become more obvious when the difference between the frequency of the primary and secondary regions increases. To avoid this problem, interpolated values of the displacements for the nodes of secondary regions are used in both independent explicit equations and (7) which is for the semi-implicit and static cases. Figure 4 demonstrates the effect of interpolation in an extreme case in which there is a large difference between the frequencies of the two regions.

The dark red nodes represent the primary region with a frequency of $1 \mathrm{kHz}$ and the light green ones represent the secondary region 
with $100 \mathrm{~Hz}$ frequency. After an input is applied, the nodes of the secondary region will not move until the end of the secondary region's time step. When the displacements of the secondary region are updated at $t_{2}$, there will be a force jump because of the sudden change in the displacements of the secondary region nodes. To achieve continuity, instead of the evaluated displacements of the secondary region being updated with $100 \mathrm{~Hz}$, interpolated values between the time steps are used in the solution of the equations. A sudden force jump occurs at time $t_{2}$ if the displacements evaluated at time $t_{1}$ are used instead of the interpolated displacements(dashed lines in figure 4) for the secondary region's nodes. Since two values have to be known for interpolation but the displacements for the next time step are not known, interpolation is applied by using the values from the previous time step. For instance, between $t_{1}$ and $t_{2}$ the interpolation algorithm uses the displacements of the secondary region evaluated at $t_{0}$ and at $t_{1}$. However this issue results in a delay effect which grows according to the frequency difference between the secondary and primary regions. This issue, however, does not prevent the use of asynchronous regions but does constrain the frequency of the secondary regions to be above a certain value.

\subsection{Analysis}

When an input is applied, the effect of the input on the displacements propagates through the model from the contact node. The model behaves like a low pass filter on the applied input signal such that if the frequency of the input is high and the frequency of the secondary regions is low, the propogation effect of the displacements diminishes before the nodes of the secondary regions are evaluated. Therefore the changes in the displacements of the nodes in the lower frequency regions become insignificant and the artificial extra force feedback grows larger for high frequency inputs. This defect depends mainly on how much lower the frequency of the secondary regions is compared with the frequency of the applied input, together with the size of the primary region. Fortunately real applications like surgery simulation provide an upper frequency limit which a human being can apply to a soft tissue [Fischer et al. 1990]. This limit can be used to determine acceptable frequency rates for the secondary regions.

\subsection{Adaptable Regions}

While the effect of the input propagates through the whole model, the number of nodes affected and the magnitude of the effect depend on the size of deformation. To achieve the best performance the size of the primary aynchronous region, the equations of which are evaluated with the highest frequency, should not be larger than absolutely necessary. The optimum size of the region is the minimum which will not make a significant difference to the feedback force, and depends on the size of the deformation, the stiffness of the tissue and the frequency of the secondary region.

To increase the efficiency, the asyncronous regions are implemented such that their sizes can be varied using a function during the simulation. The function evaluates the size of the primary region by using the size of the current deformation. Different functions can be used depending on the properties of the tissue. The use of a linear function, the slope of which can be adjusted depending on the stiffness properties of the tissue is illustrated in figure 5 for 1D. During the simulation, the deformation $\operatorname{size}\left(l_{d}\right)$ is scaled with the gradient(S) of the function. The adapted number of nodes of the primary region is calculated as the corresponding value to this scaled length(in meters) in the undeformed state. This adapted primary region size, which changes dynamically in real-time, is bounded

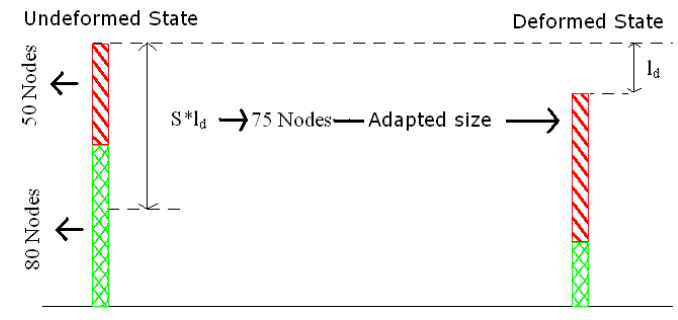

Figure 5: The use of linear function for the evaluation of adapted region size. The deformation $\operatorname{size}\left(l_{d}\right)$ is scaled with the gradient( $\left.\mathrm{S}\right)$ of the function to be used in undeformed state.

below and above by minimum and maximum node numbers specified depending on the properties of the tissue and the total number of nodes.

In the simulation only a linear function is used, however other alternatives are possible. A non-linear function could also be considered for soft tissues which tend to have a saturated deformation when the size of the deformation grows larger. Another possible way to change the size of the primary region is to use a threshold for the displacement of the nodes such that the nodes with a displacement larger than the threshold are included in the primary region. The function is therefore used to relate between deformation size and the size of the region can be adjusted experimentally for tissues with different properties. The following section includes the experiments showing the effect of the adaptable regions with linear functions, while the effect of the various functions are to be examined in future studies.

\section{Results}

Experiments have been carried out to explore the different behaviours of the asynchronous regions for changing force input appplied to different types of tissues. A one dimensional model is used to permit a number of nodes which can be solved analytically at $1 \mathrm{kHz}$ and allow a wide range of different region sizes. To be able to observe the output response objectively, a force signal is applied to the model by using a simulated haptic device, the position of which is not affected by the reaction force. The position of the haptic probe and output force are recorded and illustrated by thick red and thin blue lines, respectively, in the figures presented throughout this section. Since there is almost no response difference when the frequency of the secondary regions is not extremely low, the results of the experiments using implicit dynamic solvers are presented to emphasize the output difference for extremely low frequencies which are out of the range of explicit dynamic solvers. Direct Gaussian Elimination is used in the implicit solvers to obtain the exact result.

The parameters of the model are set to values within the range of soft tissue properties. The studies [Egorov et al. 2008; Li et al. 2006; Yu et al. 2006; Sinkus et al. 2000] on the elastic properties of soft tissue reveal that soft tissue shows complex visco-elastic and non-linear characteristics. The elasticity values measured in the different studies are: between 5 and $35 \mathrm{kPa}$ in [Egorov et al. 2008], 6 and $11 \mathrm{kPa}$ in [Li et al. 2006], 0 and $3.5 \mathrm{kPa}$ in [Sinkus et al. 2000] and $9.3 \mathrm{kPa}$ in [Yu et al. 2006]. The difference in the mentioned studies is related to tissue type, the magnitude of deformation, the non-linear behaviour of the elasticity and the level of precompression as discussed in [Samani et al. 2003]. The experiments presented in the results section of this paper have been carried out for different elasticity values between $5 \mathrm{kPa}$ and $25 \mathrm{kPa}$. The area and 


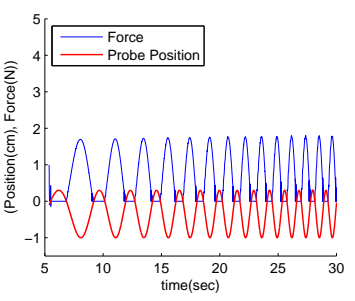

(a) $\mathrm{N}=30, \mathrm{f}=50$

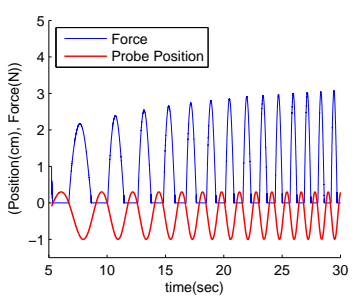

(c) $\mathrm{N}=5, \mathrm{f}=50$

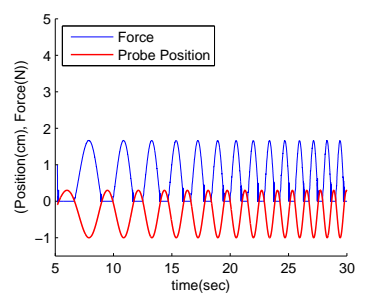

(b) $\mathrm{N}=30, \mathrm{f}=500$

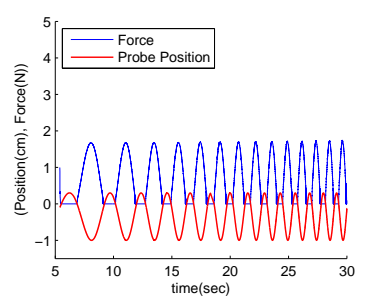

(d) $\mathrm{N}=5, \mathrm{f}=500$

Figure 6: The asyncronous solutions of the model with elasticity value of $5 \mathrm{kPa}$. $\mathrm{N}$ is the number of nodes in the primary region and $\mathrm{f}$ is the frequency of the secondary region in sub-figures.

length of the model are set to $40 \mathrm{~cm}^{2}$ and $10 \mathrm{~cm}$ respectively to keep the stiffness of the one dimensional linear model in the realistic stiffness range[Li et al. 2006] for soft tissues. The mass density is set to $1 \mathrm{gr} / \mathrm{cm}^{3}$ for soft tissue as described in [Johns and Yaffe 1987]. To achieve stability in the system, a damping value of 6.5 has been found to be optimal with these tissue properties. The total number of values is set to 40 for the model.

In the first type of experiment a chirp function, which is a sinusoid with constant magnitude and varying frequency, is applied to the model by increasing the frequency linearly. The magnitude of the deformation is kept as $1 \mathrm{~cm}$ and the frequency is increased from 0.1 to $1 \mathrm{~Hz}$. The responses of the model are compared by changing the size of the primary region and the frequency of the secondary region.

When figure 6(c) is compared to 6(a) and 6(d), it is possible to state that the output force increases slightly with the increasing input frequency in 6(c) when both the primary region's(N) size and the secondary regions' frequency(f) have lower values while there is no significant force increase in $6(\mathrm{a})$ and $6(\mathrm{~d})$ when either one of $\mathrm{N}$ or $\mathrm{f}$ has higher values.

To compare the results of asynchronous regions(figure 6) with the whole solution with $1 \mathrm{kHz}$ (figure 7(a)) on the model with elasticity value of $5 \mathrm{kPa}$, the peak values of the forces at approximately 20th second in the graphs are presented together with the solution times in table 1 . The force error and speed gain increase with the decreasing primary region size and secondary regions' frequency seperately. However when the $(\mathrm{N}=30, \mathrm{f}=50)$ and $(\mathrm{N}=5, \mathrm{f}=500)$ entries are compared, it is observed that the primary region's size has a more significant effect than the secondary regions' frequency. To exploit the speed gain of asynchronous regions the force error should be within acceptable limits throughout the simulation.

To achieve a compromise between the accuracy of the force output and speed, the size and the frequency of the regions should be adjusted properly keeping the force error within acceptable limits. If the results of the asynchronous regions with smallest primary region size and lowest secondary region frequency (figures 7(c)and

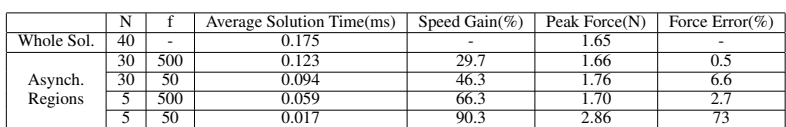

Table 1: The performance and force error comparison of the asynchronous regions with the whole analytical solution. $\mathrm{N}$ is the number of nodes solved at $1 \mathrm{kHz}, \mathrm{f}$ is the frequency of the secondary regions.

7(d)) are compared to the whole model solutions(figures 7(a) and 7(b)), one can state that the softer the tissue is, the larger the difference between the output forces of the asynchronous regions and the whole model. Therefore no optimized values have yet been found for the size of the primary region and the frequency of the secondary regions since the force output and speed depend on tissue properties and the computation power respectively. However the adaptibility feature is added to the asynchronous regions allowing changing primary region size depending on the size of the deformation in real-time. The ultimate aim in the future studies is to integrate a real-time adaptation scheme for the primary region size independent of material properties like elasticity.

\begin{tabular}{|c|c|c|}
\hline & Peak Force(N) & Force Error(\%) \\
\hline Whole Sol. & 24.96 & - \\
\hline Adaptible a.r & 25.93 & 3.9 \\
\hline Non-Adaptible a.r & 31.07 & 24.5 \\
\hline
\end{tabular}

Table 2: The performance and force error comparison of the adaptible and non-adaptible asynchronous regions with the whole analytical solution.The Peak Force is the value of the force at approximately 30th second of the graphs in figure 8 .

In the second set of experiments, the behaviour of the adaptable primary region size is examined by changing the size depending on the deformation length in real-time. A force input with constant frequency and increasing magnitude $(\max 5 \mathrm{~cm}$.) is applied to the model. Figure 8(a) illustrates the difference between the solution of the whole model with $1 \mathrm{kHz}$ and solution of non-adaptable and adaptable asynchronous regions with a primary region size of 10 and secondary region frequency of $50 \mathrm{~Hz}$. A linear function with a gradient of 4 is used for the adaptable region in figure 8(b) which means the ratio of the length of the primary region to the deformation length is 4 . However in order not to solve the whole model with the highest frequency, there should be a limit for the maximum size of the primary region which is 30 nodes in this case. The parameters are chosen empirically to show only the benefit of using adaptible regions over non-adaptible regions and have not been optimized yet. The force error difference between adaptible and nonadaptible regions can be examined from table 2, while from figure 8 it can also be observed that the force error increases with increasing deformation size proving the necessity to consider the deformation size while adapting the size of the primary region. The maximum size of the primary region is, of course, ultimately constrained by the available computational power.

\section{Conclusions and Future Work}

In this study, in order to attain sufficient refresh rates for the solution of FEM equations in real-time surgery simulations, asynchronous regions are proposed. The FEM equations are separated into different groups, the equations of each are solved with different frequencies. In the event of deformation it is then possible to update the displacements of the nodes in the local neighbourhood of the contact point at sufficient frequency to provide a realistic 


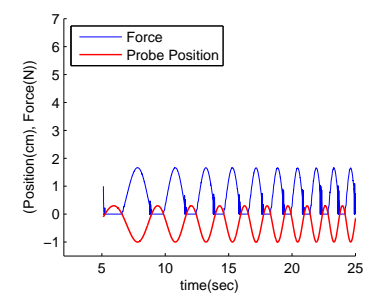

(a) $\mathrm{E}=5 \mathrm{kPa}$

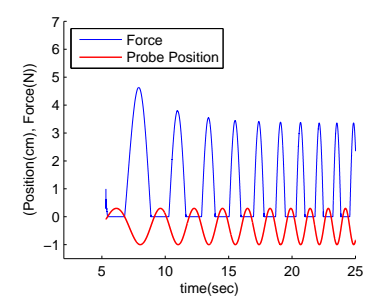

(c) $\mathrm{E}=5 \mathrm{kPa}$

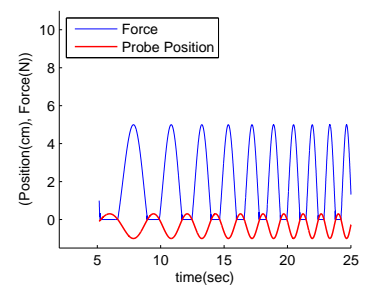

(b) $\mathrm{E}=25 \mathrm{kPa}$

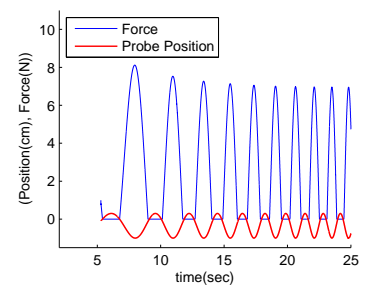

(d) $\mathrm{E}=25 \mathrm{kPa}$
Figure 7: The solution of the whole model with $1 \mathrm{kHz}(7(\mathrm{a}), 7(\mathrm{~b}))$ and the solution by asynchronous regions with a primary region size of 5 nodes and $5 \mathrm{~Hz}$ frequency of secondary regions(7(c),7(d).

simulation, while updating the more remote regions with a lower frequency. The computational power saved by solving the more distant regions at lower frequencies can then be dedicated to modelling the complex behaviours of soft tissue in the local region, like visco-elasticity, anisotropicity and non-linearity.

Static, implicit dynamic and explicit dynamic solution methods have been implemented to solve the FEM equations. Although the explicit dynamic solvers provide better performance and permit easier updating of the model, for example in the case of tissue cutting, these solvers are only conditionally stable such that a high refresh rate is needed for the solutions to converge. By using asynchronous regions it is possible to solve different regions not only at different frequencies but also to apply different solution methods. To simulate the tissue cutting it is possible to exploit this feature of the asynchronous regions by solving the local neighborhood of the contact by explicit dynamic solvers with high refresh rates and the other regions using unconditionally stable solvers with lower frequencies.

The behaviour of the asynchronous regions has been tested through comparison with an analytically solved simulation in a one dimensional linear model by applying different input signals to different tissue types. From the results of the experiments, one can state that when the size of the primary region decreases and the refresh rate of the secondary regions is reduced, the difference between the output forces of asynchronous regions and the whole model increases, while increasing the primary region size and secondary regions' frequency results in a smaller force difference as expected. However to preserve a $1 \mathrm{kHz}$ haptic refresh rate, with an acceptable output force error, the primary region size and secondary regions' frequencies should not be more than necessary. Therefore to exploit the method of asynchronous regions without having a significant error in the force, the size of the primary region and the frequency of the secondary region should be optimized which will be explored in the future studies. The performance measurements have also shown that it is possible to achieve significant improvement in the calculation speed while keeping the force error in acceptable ranges.

To obtain maximum efficiency adaptable asynchronous regions are

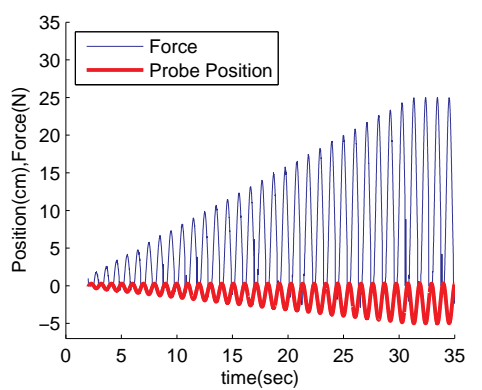

(a) Whole solution

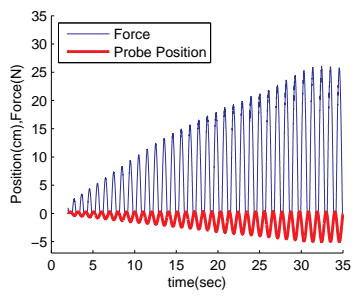

(b) Adaptable region

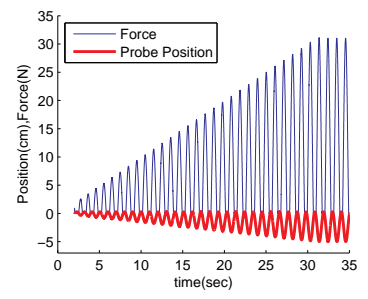

(c) Non-adaptable region
Figure 8: Solution of the whole method with $1 \mathrm{kHz}$, non-adaptable asynchronous regions, and adaptable regions.

described, the size of which can be varied in real-time depending on the deformation applied. The size of the primary region is related to the deformation by using a linear function and it is limited to a maximum value. To achieve best performance the gradient of the linear function and the maximum limit can be adjusted depending on the complexity of the model and the available computation power. It is observed that adaptable regions provide better results than non-adaptable regions especially for high magnitude inputs.

As a future study, the relationship between the function used to adapt the size of the regions and tissue properties can be examined to obtain material property independent function. Evaluation of asynchronous regions, integration of level of detail in the spatial domain, and non-linearity are also points to be explored.

\section{Acknowledgements}

This work has been funded by the Swedish Science Council through grant number 621-2005-3609 and by the Foundation for Strategic Research (SSF) under the Strategic Research Center MOVIII.

\section{References}

Astley, O., AND HAYWARD, V. 1998. Multirate haptic simulation achieved by coupling finite element meshes through norton equivalents. In Robotics and Automation, 1998. Proceedings. 1998 IEEE International Conference, 989-994 vol.2.

Barbagli, F., SAlisbury, K., And Prattichizzo, D. 2003. Dynamic local models for stable multi-contact haptic interaction with deformable objects. In Haptic Interfaces for Virtual Environment and Teleoperator Systems, 2003. HAPTICS 2003. Proceedings. 11th Symposium, 109-116. 
BAthe, K.-J. 1982. Finite Element Procedures in Engineering Analysis. Prentice Hall.

Bro-Nielsen, M., AND Cotin, S. 1996. Real-time volumetric deformable models for surgery simulation using finite elemets and condensation. In Computer Graphics Forum, 57-66.

Cavusoglu, M., And Tendick, F. 2000. Multirate simulation for high fidelity haptic interaction with deformable objects in virtual environments. In Robotics and Automation, 2000. Proceedings. 2000 IEEE International Conference, 2458-2465.

Cotin, S., Delingette, H., And Ayache, N. 1999. Real-time elastic deformations of soft tissues for surgery simulation. IEEE Transactions on Visualization and Computer Graphics, 62-73.

Debunne, G., Desbrun, M., Cani, M., And Barr, A. 2001. Dynamic real-time deformations using space \& time adaptive sampling. In SIGGRAPH '01: Proceedings of the 28th annual conference on Computer graphics and interactive techniques, 31-36.

Delingette, H., Cotin, S., And Ayache, N. 1999. A hybrid elastic model allowing real-time cutting, deformations and force-feedback for surgery training and simulation. In $C A$ ' 99 : Proceedings of the Computer Animation.

Egorov, V., Tsyuryupa, S., Kanilo, S., Kogit, M., And A Sarvazyan. 2008. Soft tissue elastometer. Medical Engineering and Physics, 206-212.

Fischer, P., DANIEL, R., AND SIVA, K. 1990. Specification and design of input devices for teleoperation. Robotics and Automation, 1990. Proceedings., 1990 IEEE International Conference on, 540-545 vol.1.

Frank, A. O., Twombly, I. A., Barth, T. J., And Smith, J. D. 2001. Finite element methods for real-time haptic feedback of soft-tissue models in virtual reality simulators. In $V R$ '01: Proceedings of the Virtual Reality 2001 Conference (VR'01), 257-263.

Gosline, A. H., Salcudean, S. E., And Yan, J. 2004. Haptic simulation of linear elastic media with fluid inclusions, hapticse: Electronic. Journal of Haptics Research 3.

Heng, P.-A., Cheng, C.-Y., Wong, T.-T., Xu, Y., Chui, Y.P., Chan, K.-M., AND Tso, S.-K. 2004. A virtual-reality training system for knee arthroscopic surgery. IEEE Transactions on Information Technology in Biomedicine, 217-227.

JeŘÁb Ková, L., Kuhlen, T., Wolter, T. P., And Pallua, N. 2004. A voxel based multiresolution technique for soft tissue deformation. In VRST '04: Proceedings of the ACM symposium on Virtual reality software and technology, 158-161.

Johns, P. C., AND YAFFE, M. J. 1987. X-ray characterisation of normal and neoplastic breast tissues. Physics in Medicine Biology, 675-695.

Li, X., Wang, G., HuAng, L., And Zhang, G. 2006. Young's modulus extraction methods for soft tissue from ultrasound measurement system. Instrumentation Science and Technology, 393404.

Lindblad, A., AND TURKiYyah, G. 2007. A physically-based framework for real-time haptic cutting and interaction with $3 \mathrm{~d}$ continuum models. In SPM '07: Proceedings of the 2007 ACM symposium on Solid and physical modeling, 421-429.
Maurel, W., Wu, Y., Thalmann, N. M., and Thalmann, D. 1998. Biomechanical Models for Soft Tissue Simulation. Springer-Verlag.

Mor, A. B., AND Kanade, T. 2000. Modifying soft tissue models: Progressive cutting with minimal new element creation. In In MICCAI, 598-608.

Nienhuys, H.-W., And VAn Der Stappen, A. F. 2000. A Surgery Simulation Supporting Cuts and Finite Element Deformation. Springer Berlin / Heidelberg, 145-152.

Picinbono, G., Lombardo, J. C., Delingette, H., And AYACHE, N. 2000. Anisotropic elasticity and force extrapolation to improve realism of surgery simulation. In $n$ IEEE International Conference on Robotics and Automotion: ICRA 2000, 596-602.

Picinbono, G., Delingette, H., And Ayache, N. 2003. Non-linear anisotropic elasticity for real-time surgery simulation. Graph. Models, 305-321.

Samani, A., Bishop, J., Luginbuhl, C., And Plewes, D. B. 2003. Measuring the elastic modulus of ex vivo small tissue samples. Physics in Medicine Biology, 2183-2198.

Sedef, M., Samur, E., And Basdogan, C. 2006. Real-time finite-element simulation of linear viscoelastic tissue behavior based on experimental data. IEEE Computer Graphics and Applications, 58-68.

Sela, G., Subag, J., Lindblad, A., Albocher, D., Schein, S., AND ELBER, G. 2007. Real-time haptic incision simulation using fem-based discontinuous free-form deformation. Comput. Aided Des., 685-693.

Sinkus, R., Lorenzen, J., Schrader, D., Lorenzen, M., DARGATZ, M., AND Holz, D. 2000. High-resolution tensor mr elastography for breast tumour detection. Physics in Medicine Biology, 1649-1664.

Vigneron, L. M., Verly, J. G., ANd Warfield, S. K. 2004. Modelling Surgical Cuts, Retractions, and Resections Via Extended Finite Element Method. Springer Berlin / Heidelberg, 311-318.

Wu, W., AND Heng, P. A. 2005. An improved scheme of interactive finite element model for $3 \mathrm{~d}$ soft tissue cutting and deformation. The Visual Computer, 707-716.

Wu, X., Downes, M. S., Goktekin, T., And Tendick, F. 2001. Adaptive nonlinear finite elements for deformable body simulation using dynamic progressive meshes. In Computer Graphics Forum, 349-358.

Yan, Z., Gu, L., Huang, P., Lv, S., Yu, X., And Kong, X. 2007. Soft tissue deformation simulation in virtual surgery using nonlinear finite element method. In Engineering in Medicine and Biology Society, 2007 EMBS.29th Annual International Conference of the IEEE, 3642-3645.

Yu, W., LI, Y., ZHENG, Y. P., LIM, N. Y., LU, M. H., AND FAN, J. 2006. Softness measurements for open-cell foam materials and human soft tissue. Measurement Science and Technology, $1785-1791$.

ZHUANG, Y., AND CANNY, J. 2000. Haptic interaction with global deformations. In Robotics and Automation, 2000. Proceedings. ICRA 'O0. IEEE International Conference, 2428-2433.

Zienkiewicz, O. C., TAYlor, R. L., AND Zhu, J. Z. 2005. The Finite Element Method: Its Basis and Fundamentals. Elsevier Butterworth Heinemann, Oxford. 